\title{
Outcome of Closed and Open Reduction with Cross Pinning Fixation for Displaced Supracondylar Humeral Fracture
}

\author{
Megaputera, H Suroto, D Roeshadi \\ Department of Orthopaedics \& Traumatology, Dr Soetomo General Hospital, Surabaya, Indonesia
}

\begin{abstract}
Introduction: In areas where intraoperative radiography facilities are not available, open reduction is an acceptable treatment option for fracture treatment. The aim of this investigation was to compare the outcomes of closed reduction to open reduction with cross-pinning fixation in Gartland Type III supracondylar humeral fracture extensions. Methods: In this retrospective study, closed reduction with cross pinning fixation was performed in 7 patients with closed fractures, and open reduction with cross pinning fixation was performed in 7 patients with open fractures. Result: There was no significant difference between closed and open reduction with cross pinning fixation in Gartland Type III supracondylar humeral fracture extensions in terms of postoperative carrying angle and join range of motion. $(p>0.05)$. There were no wound related complications in either group. Conclusion: Open reduction with cross pinning fixation is a viable treatment option for Gartland type III supracondylar humeral fracture extensions especially when there are no intra-operative radiography facilities $(\mathrm{p}>0.05)$.
\end{abstract}

Key Words:

Cross pinning, Open Reduction, Supracondylar Humeral Fracture

\section{INTRODUCTION}

Supracondylar fractures of the humerus are the most common type of elbow fracture in children. Controversy exists regarding the ideal method for treatment of displaced supracondylar humeral fractures. Recommended treatment modalities vary from no reduction and with immobilisation to open reduction and internal fixation. Due to the difficulty involved in maintenance of adequate reduction with cast immobilisation, fixation of reduced fractures with pins placed percutaneously has become the universally accepted method of treatment ${ }^{1,2}$.

There have been numerous variations in pinning techniques. The risk of iatrogenic ulnar nerve injury is always a concern during insertion of the medial pin. The aim of the present study is to evaluate the results of closed and open reduction with cross pinning fixation in Gartland Type III supracondylar humeral fracture extensions.

\section{MATERIALS AND METHODS}

Between July 2007 and July 2009, 14 children with Gartland Type III supracondylar humeral fracture extensions were managed with closed or open reduction, both with cross pinning fixation in our institution. The study cohort includes 8 boys $(57.1 \%)$ and 6 girls $(42.9 \%)$ with an age range from 6 to $8 y$. Most injuries were due to falling during running $(60 \%)$. On presentation, patients underwent thorough clinical assessment with special attention to peripheral circulation and neurological status.

Closed reductions of fractures (utilizing an image intensifier) were performed in 7 patients $(50 \%)$. Open reductions of fractures were performed in 7 patients $(50 \%)$ with grade I open fracture according to Gustillo classification. All patients underwent fracture within $24 \mathrm{~h}$ of the trauma. A Kwire of $1.6 \mathrm{~mm}$ diameter was used in all cases; the direction of placement for the first wire was from inferomedial to superolateral, in order to preserve the ulnar nerve. The second wire was directed from inferolateral to superomedial. All operations were performed by the same surgeon.

Care was taken in postoperative neurological assessment of median, ulnar, and radial nerves function. Period of hospitalisation was 1-2 days. Patients were followed up 1 week postoperatively for radiological confirmation of reduction maintenance, at 4 weeks to remove the $\mathrm{K}$ wires and initiate joint movement, and subsequently monthly for a minimum of 4 months. At the last follow-up, patients were assessed both radiological for union and functionally according to Flynn's criteria (Table I) ${ }^{3}$. Data analysis was performed utilizing SPSS software (version 10) and included calculation of the arithmetic mean and standard deviation as quantitative variables. Comparison of postoperative carrying angle and range of motion were performed using student's ttest. 
Table I: Flynn's criteria for evaluating physical outcome of supracondylar fracture of humerus ${ }^{3}$

\begin{tabular}{|lccl|}
\hline & $\begin{array}{c}\text { Cosmetic factor } \\
\text { carrying-angle loss (degrees) }\end{array}$ & $\begin{array}{c}\text { Functional factor } \\
\text { movement loss (degrees) }\end{array}$ & Overall rating \\
\hline Excellent & 0 to 5 & 0 to 5 & The lower of the two ratings, \\
Good & 5 to 10 & 5 to 10 & and an elbow with a varus \\
Fair & 10 to 15 & 10 to 15 & deformity is automatically \\
Poor & $>15$ & $>15$ & graded as poor \\
\hline
\end{tabular}

Table II: Comparison between children treated with open and closed reduction

\begin{tabular}{|lcccc|}
\hline & $\begin{array}{c}\text { Mean carrying } \\
\text { angle (degrees) }\end{array}$ & Difference * & $\begin{array}{c}\text { Mean range } \\
\text { of motion (degrees) }\end{array}$ & Difference * \\
\hline Open reduction & 12.6 & 2.43 & 142.3 & 2.71 \\
Closed reduction & 12.3 & 2.72 & 142.3 & 2.71 \\
\hline
\end{tabular}

* mean of the difference between treated side and opposite normal side

\section{RESULTS}

There was no incidence of pin tract infection in both groups. For the open fracture cases that were treated with open reduction, there were no superficial or deep infection recorded. Patients did not complain about the scar. All study subjects had full range of the elbow motion postoperatively compared to the contralateral (non-injured) side, and the mean postoperative carrying angle of the injured elbow was $15^{\circ}$ (range $10^{\circ}-20^{\circ}$ ). There were no known preoperative median, radial or ulnar nerve injuries in the study cohort, and no iatrogenic nerve injuries were observed during follow-up. Based on Flynn's criteria, all children had satisfactory cosmetic and functional results.

Differences between carrying angle and range of motion between treated and the opposite elbow were evaluated. When we compared the values between children treated with open and closed reduction, there was no statistical difference between the 2 groups in term of carrying angle $(p=0.718)$ and range of motion $(\mathrm{p}=1.00)$. For both the groups, cosmetic and functional outcome were excellent (Table II).

\section{DISCUSSION}

Supracondylar fracture of the humerus is the most common type of elbow fracture in children and adolescents. It accounts for $50-70 \%$ of elbow fractures and is seen most frequently in children between the age of 3 and 10 years (Blount) There exists controversy about the optimal treatment modality for displaced supracondylar fractures. Several treatment modalities have been recommended including closed reduction and plaster immobilisation, open reduction and internal fixation, traction and closed reduction, and percutaneous pinning ${ }^{4,5,6}$. While closed manipulation and percutaneous K-wire stabilization is widely used treatment for this condition, there is no general consensus about optimal configuration of the K-wires. The two-wire crossfixation is frequently used and good results have been reported ${ }^{2,7}$.
In our small series there was not a significant difference between patients treated with closed and open reduction in any measured parameters. Considering the fact that all children treated with open reduction sustained open fractures, we would expect higher energy trauma and more severe soft tissue injury (from both the trauma and the surgical approach) in this group. However, the short-term physical outcomes appear to be comparable. In the case of open fractures, there is no question about the need to expose the fracture site, but we are interested in the outcomes of this treatment method and suggest its use as reference for use with closed fractures in developing countries. In areas with no intraoperative radiography facilities, orthopaedists must weigh the risk of malunion from closed reduction without fixation compared to additional surgical scarring inherent from open reduction and wire fixation. It seems that the main differences between the two procedures are related to cosmetic outcome. Many hospitals around the world especially in the developing countries do not have intraoperative radiography, and the outcome of this study supports the use of open reduction followed by wire fixation as one treatment option for Gartland Type III.

\section{CONCLUSION}

This retrospective study shows that there is no significant difference in carrying angle and range of motion following treatment with closed and open reduction for Gartland type III supracondylar humeral fractures. While open reduction should be considered for all open fractures, this treatment option should be used for closed fractures when no intraoperative radiography facility is available. 


\section{REFERENCES}

1. Alcott WH, Bowden BW, Miller PR. Displaced supracondylar fractures of the humerus in children: long-term follow-up of 69 patients. J Am Osteopath Assoc. 1977: 76: 910-915.

2. Wilkins KE. Fractures and dislocations of the elbow region, in Rockwood CA Jr, Wilkins KE, King RE (eds): Fractures in Children, 3rd edition.

3. Flynn JC, Matthews JG, and Benoit RL. Blind pinning of displaced supracondylar fractures of the humerus in children. $J$ Bone Joint Surg Am. 1974: 56: 263-272.

4. Worlock PH, Colton CL. Displaced supracondylar fractures of the humerus in children treated by overhead olecranon traction. Injury. 1984; 15: 316-21

5. Topping RE, Blanco JS, David TJ. Clinical evaluation of crossed-pin versus lateral-pin fixation in displaced supracondylarhumerus fractures. J Pediatr Orthop. 1995; 15: 435-439.

6. Foead A, Penafort R, Saw A, Sengupta S. Comparison of two methods of percutaneous pin fixation in displaced supracondylar fractures of the humerus in children. J Orthop Surg. 2004; 12: 76-82.

7. Minkowitz B, Busch MT: Supracondylar humerus fractures: Current trends and controversies. Orthop Clin North Am. 1994; 25; 581-594 